\title{
Improved Estimation of Pollutant Emission Rate in an Ocean Pollutant Diffusion Model by the Application of Spline Interpolation with the Adjoint Method
}

\author{
XiaOlOng Zong AND HAidong PAN \\ Key Laboratory of Physical Oceanography, Ocean University of China, and Qingdao National Laboratory for Marine Science \\ and Technology, Qingdao, China \\ YONGZHI LIU \\ Key Laboratory of Physical Oceanography, Ocean University of China, and Qingdao National Laboratory for Marine Science \\ and Technology, and The First Institute of Oceanography, State Oceanic Administration, Qingdao, China \\ XIANQING LV \\ Key Laboratory of Physical Oceanography, Ocean University of China, and Qingdao National Laboratory for Marine Science \\ and Technology, Qingdao, China
}

(Manuscript received 4 December 2017, in final form 29 July 2018)

\begin{abstract}
The spline interpolation method is applied to the inversion of the time-varying pollutant emission rate based on an ocean pollutant diffusion model with the adjoint method. A series of numerical experiments are performed to compare the spline interpolation with the Cressman interpolation. Experimental results show that the spline interpolation improves the inversion results in terms of the smoothness and accuracy. Furthermore, it is the advantages of spline interpolation-better resistance to the impact of errors and demand for fewer observations- that give rise to a better performance in practice.
\end{abstract}

\section{Introduction}

Human economic activities have had severe impact on marine ecosystems (Øyvind et al. 2003; Eyring et al. 2005; Correa et al. 2015; Meissa and Gascuel 2015; Xing et al. 2015; Sidorovskaia et al. 2016). Among these, the accidental release of pollutants into the ocean poses a serious threat to marine ecosystems, such as crude oil leakage (Snowden and Ekweozor 1987; Walsh et al. 2015, 2016; Wei et al. 2015) and release of hazardous materials (Zhang et al. 2013; Zhang and Huang 2017). For these incidents, a crucial aspect within the evaluation of risks and the remediation process is to identify the accurate locations of pollutant sources and to understand the release histories that led to the pollution (Milnes and Perrochet 2007). This is significant for assisting decision makers to effectively plan countermeasures.

The issue of identification of pollutant source location has been extensively investigated over the past decades (Neupauer and Wilson 2005; Neupauer and Lin 2006;

Corresponding author: Xianqing Lv, xqinglv@ouc.edu.cn
Datta et al. 2011). Simultaneously, the reconstruction of pollutant release history has also attracted much attention. Atmadja and Bagtzoglou (2001) first used the backward beam equation method to solve the advectiondispersion equation with heterogeneous parameters and this method was able to recover the release history roughly. Later a new variant of robust least squares, the constrained robust least squares, was introduced to reconstruct pollutant release history by Sun et al. (2006). Zanini and Woodbury (2016) presented a new approach, an empirical Bayesian method combined with Akaike's Bayesian information criterion, to estimate the pollutant release history. These efforts were mainly focused on the pollution in groundwater, yet we are immersed in the reconstruction of pollutant release history in ocean based on the adjoint assimilation method in this work.

Essentially, the reconstruction of pollutant release history based on limited observations is an inverse problem (Sun et al. 2006). Many works have proved that the adjoint assimilation method is adept in solving this sort of problems (Yang and Zabaras 1998; Oberai et al. 2003; Michalak and Kitanidis 2004; Z. Chen et al. 2014; 
Shen et al. 2015). There are two major advantages, the numerical stability and the reductions of simulation runs, of the adjoint assimilation method when coupled with numerical models (Milnes and Perrochet 2007). Neupauer and Wilson (1999) applied the adjoint method to obtaining backward location and travel time probabilities, which could be used to determine the prior location of contamination in an aquifer. Wang et al. (2013) inverted the initial field of pollutants by assimilating routine monitoring databased on the adjoint assimilation method. Liu et al. (2017) applied the adjoint assimilation method to inverting the prescribed spatiotemporally distributed nonpoint source terms and initial values of total nitrogen.

However, the inverse problem is often beset by illposedness (Smedstad and O'Brien 1991; Navon 1998). The independent point scheme (IPS), serving as an effective solution, is an efficient method for reconstructing the time-varying pollutant emission rate of a known point source in ocean. In the IPS the values of model parameters at some selected points, called independent points, are treated as independent variables and the values of model parameters at other points are obtained by interpolating the independent variables (Guo et al. 2017). In this work, the independent points refer to some selected time points during the simulation period. The IPS is a valid approach to reduce the quantity of variables that need to be inverted and promote the efficiency of the adjoint assimilation model. Based on the IPS, Lu and Zhang (2006) estimated spatially varying bottom friction coefficient for a $2 \mathrm{D}$ tidal model; Li et al. (2013) verified that the spatiotemporal parameters could improve the simulation precision for a marine ecosystem dynamical model; and $\mathrm{H}$. Chen et al. (2014) estimated the spatially varying open boundary conditions for a numerical internal tidal model. As an improvement, the above scheme is modified in this work, in which the Cressman interpolation (CI) is replaced by the cubic spline interpolation (SI).

This paper is organized as follows. Section 2 introduces the numerical models and interpolation methods. Model settings and numerical experiments are conducted in section 3. Section 4 discusses the impact of error and number of observations on inversion results. Finally, conclusions are presented in section 5.

\section{Methodology}

\section{a. The ocean pollutant diffusion model}

Advection and diffusion are major physical mechanisms for the transportation process of conservative ocean pollutants. As a result, the basic advection and diffusion equation is selected as a model equation, and an item that depicts the emission rate of the pollutant is added as a modification, which is written as follows based on a hydrodynamic background field:

$$
\begin{aligned}
\frac{\partial \mathbf{C}}{\partial t} & +u \frac{\partial \mathbf{C}}{\partial x}+v \frac{\partial \mathbf{C}}{\partial y}+w \frac{\partial \mathbf{C}}{\partial z} \\
& =\frac{\partial}{\partial x}\left(A_{H} \frac{\partial \mathbf{C}}{\partial x}\right)+\frac{\partial}{\partial y}\left(A_{H} \frac{\partial \mathbf{C}}{\partial y}\right)+\frac{\partial}{\partial z}\left(K_{V} \frac{\partial \mathbf{C}}{\partial z}\right)+\theta(t),
\end{aligned}
$$

where $\mathbf{C}$ represents the concentration of pollutant; $t$ is the time; $x, y$, and $z$ are components of the Cartesian coordinate system; $u, v$, and $w$ are velocities in the $x, y$, and $z$ directions, respectively; $A_{H}$ and $K_{V}$ are the horizontal and vertical diffusivity coefficients, respectively; and $\theta(t)$ represents the time-varying pollutant emission rate at the point source, which could be modified according to the number and locations of the known pollutant point sources in the study area. In this work, we assume there is only one pollutant point source in the study area. The finite difference form of the model equation [Eq. (1)] is available in Wang et al. (2013).

To guarantee mass conservation, the open boundary condition is set as radiation boundary conditions:

$$
\left\{\begin{array}{l}
\frac{\partial \mathbf{C}}{\partial t}=0, \quad V_{n} \leq 0 \\
\frac{\partial \mathbf{C}}{\partial n}=0, \quad V_{n}>0
\end{array},\right.
$$

where $n$ represents the normal directions of open boundary and $V_{n}$ is the flow speed of seawater in the normal direction of open boundary.

\section{b. The adjoint model}

To construct the adjoint model, the cost function $J$, the distance between simulations and observations, is defined as

$$
J(\mathbf{C})=\frac{1}{2} \int_{\Omega} \mathbf{K}_{C}(\mathbf{C}-\overline{\mathbf{C}})^{\mathrm{T}}(\mathbf{C}-\overline{\mathbf{C}}) d \Omega,
$$

where $\mathbf{C}$ and $\overline{\mathbf{C}}$ are the simulated and the observed pollutant concentrations, respectively. Also, $\mathrm{T}$ denotes matrix transposition, $\Omega$ is the model domain, and $\mathbf{K}_{C}$ is the weighting matrix, which should theoretically be the inverse of the observational error covariance matrix. But it can be simplified by assuming that the errors of the data are uncorrelated and equally weighted (Yu and O'Brien 1992). In this work, the element of $\mathbf{K}_{C}$ is 1 where observations are available, and 0 otherwise (Liu et al. 2017).

Based on the Lagrangian multiplier method, the Lagrangian function is defined as 


$$
\begin{aligned}
L\left(\mathbf{C}^{*}, \mathbf{C}\right)= & \int_{\Omega} \mathbf{C}^{*}\left\{\frac{\partial \mathbf{C}}{\partial t}+u \frac{\partial \mathbf{C}}{\partial x}+v \frac{\partial \mathbf{C}}{\partial y}+w \frac{\partial \mathbf{C}}{\partial z}\right. \\
& -\frac{\partial}{\partial x}\left(A_{H} \frac{\partial \mathbf{C}}{\partial x}\right)-\frac{\partial}{\partial y}\left(A_{H} \frac{\partial \mathbf{C}}{\partial y}\right) \\
& \left.-\frac{\partial}{\partial z}\left(K_{V} \frac{\partial \mathbf{C}}{\partial z}\right)-\theta(t)\right\} d \Omega+J(\mathbf{C}),
\end{aligned}
$$

where $\mathbf{C} *$ denotes the adjoint variable of $\mathbf{C}$. The adjoint model can be constructed by the following equation:

$$
\frac{\partial L}{\partial \mathbf{C}}=0
$$

yielding the following adjoint equation:

$$
\begin{aligned}
-\frac{\partial \mathbf{C}^{*}}{\partial t} & -\frac{\partial}{\partial z}\left(K_{V} \frac{\partial \mathbf{C}^{*}}{\partial z}\right) \\
= & \frac{\partial\left(u \mathbf{C}^{*}\right)}{\partial x}+\frac{\partial\left(v \mathbf{C}^{*}\right)}{\partial y}+\frac{\partial\left(w \mathbf{C}^{*}\right)}{\partial z}+\frac{\partial}{\partial x}\left(A_{H} \frac{\partial \mathbf{C}^{*}}{\partial x}\right) \\
& +\frac{\partial}{\partial y}\left(A_{H} \frac{\partial \mathbf{C}^{*}}{\partial y}\right)-\mathbf{K}_{C}(\mathbf{C}-\overline{\mathbf{C}}) .
\end{aligned}
$$

\section{c. Interpolation methods}

Cressman (1959) introduced a weighting factor for the pressure-surface height analysis, and made a great progress for the interpolation procedure in the field of sparse data (Goodin et al. 1979). Then, this interpolation method had been widely used in many different fields (Barnes 1964; Le Dimet and Talagrand 1986; Xu et al. 1994; Zhang et al. 2014; Liu et al. 2016). In a series of previous works using the IPS, the CI was selected to interpolate the values at independent points (Zhang and Lu 2008; Fan and Lv 2009; Li et al. 2013; H. Chen et al. 2014; Shen et al. 2015). The CI could be represented as follows:

$$
\begin{gathered}
\theta_{j}=\sum_{k=1}^{N} \phi_{j, k} \times \theta_{k}, \\
\phi_{j, k}=\frac{w_{j, k}}{\sum_{k=1}^{N} w_{j, k}},
\end{gathered}
$$

within the time interval $\left[t_{i}, t_{i+1}\right]$. Then, $\theta(t)$ can be written as

where $\theta_{j}$ is the pollutant emission rate at the $j$ th time point, $\theta_{k}$ represents the pollutant emission rate at the $k$ th independent time point, $\phi_{j, k}$ is the normalized interpolation weight of the $k$ th independent time point at the $j$ th time point, $N$ is the total number of the independent time point, $w_{j, k}$ is the weighting factor, $R$ represents the influence radius, and $r_{j, k}$ is the distance between the $j$ th time point and the $k$ th independent time point.

However, the CI is a kind of linear interpolation, which causes the deviation for curve interpolation (Shen et al. 2015; Pan et al. 2017) and needs to be improved. To deal with this problem, the SI is introduced into the IPS, which usually shows better performance in producing smooth curves. Perrin et al. (1987) applied the SI to mapping scalp potentials and a higher level of smoothness and precision was shown in the comparison with linear interpolation. Unser et al. (1991) applied the SI to continuous image representation and interpolation, and they concluded that this type of interpolation method did not result in a loss of image resolution and performed in a very efficient manner. Wijnands et al. (2016) used the SI to establish a model of near-surface wind speeds in tropical cyclones and the comparison with an earlier linear wind model demonstrated its superiority. Recently, this interpolation method combined with the IPS had achieved success in inversion of the tidal boundary conditions (Pan et al. 2017) and bottom friction coefficients (Guo et al. 2017).

The SI can also be represented as Eq. (7), but the calculation of interpolation weight $\phi_{j, k}$ is more complex. Supposing that the pollutant emission rate can be represented as a cubic spline $\theta(t)$, and

$$
\left\{\begin{array}{l}
\theta\left(t_{i}\right)=e_{i}, \quad \theta\left(t_{i+1}\right)=e_{i+1}, \quad i=0,1, \ldots, N-1 \\
\theta^{\prime}\left(t_{i}\right)=m_{i}, \quad \theta^{\prime}\left(t_{i+1}\right)=m_{i+1}, \quad i=0,1, \ldots, N-1
\end{array}\right.
$$

$$
\begin{aligned}
\theta(t)= & \left(1+2 \frac{t-t_{i}}{t_{i+1}-t_{i}}\right)\left(\frac{t-t_{i+1}}{t_{i}-t_{i+1}}\right)^{2} e_{i}+\left(1+2 \frac{t-t_{i+1}}{t_{i}-t_{i+1}}\right)\left(\frac{t-t_{i}}{t_{i+1}-t_{i}}\right)^{2} e_{i+1} \\
& +\left(t-t_{i}\right)\left(\frac{t-t_{i+1}}{t_{i}-t_{i+1}}\right)^{2} m_{i}+\left(t-t_{i+1}\right)\left(\frac{t-t_{i}}{t_{i+1}-t_{i}}\right)^{2} m_{i+1}
\end{aligned}
$$


The term $\theta(t)$ is a cubic spline, so its second derivative is continuous at the time point $t_{i}$ [i.e., $\left.\theta^{\prime \prime}\left(t_{i}^{+}\right)=\theta^{\prime \prime}\left(t_{i}^{-}\right)\right]$, and it is derived as follows:

$$
\begin{aligned}
& \frac{6}{h_{i-1}^{2}} e_{i-1}-\frac{6}{h_{i-1}^{2}} e_{i}+\frac{2}{h_{i-1}} m_{i-1}+\frac{4}{h_{i-1}} m_{i} \\
& =-\frac{6}{h_{i}^{2}} e_{i}+\frac{6}{h_{i}^{2}} e_{i+1}-\frac{4}{h_{i}} m_{i}-\frac{2}{h_{i}} m_{i+1} \\
& \quad \times\left(h_{i}=t_{i+1}-t_{i}\right) .
\end{aligned}
$$

The above equation can be simplified as

$$
\left(1-\alpha_{i}\right) m_{i-1}+2 m_{i}+\alpha_{i} m_{i+1}=\beta_{i}, \quad i=1,2, \ldots, N-1,
$$

where

$$
\left\{\begin{array}{l}
\alpha_{i}=\frac{h_{i-1}}{h_{i-1}+h_{i}} \\
\beta_{i}=3\left[\frac{1-\alpha_{i}}{h_{i-1}}\left(e_{i}-e_{i-1}\right)+\frac{\alpha_{i}}{h_{i}}\left(e_{i+1}-e_{i}\right)\right]
\end{array}\right.
$$

It is similar to the assumption in Pan et al. (2017) that $h_{i}=h_{i-1}=h$ in this work. Hence, Eq. (14) can be rewritten as

$$
\alpha_{i}=\frac{1}{2}, \quad \beta_{i}=\frac{3}{2 h}\left(e_{i+1}-e_{i-1}\right) .
$$

Equation (13) is an equation set that has $N+1$ variables and $N-1$ equations, which causes infinite multisolutions. Therefore, a boundary condition is necessary. As an example, the first boundary condition is selected in this work, in which the slopes $\left(m_{0}, m_{N}\right)$ of tangent at $t_{0}, t_{N}$ are known. It is prescribed for the derivation that $m_{0}=m_{N}=1$. Therefore, Eq. (13) can be rewritten as

$$
\left\{\begin{array}{l}
m_{0}=m_{N}=1 \\
\frac{1}{2} m_{i-1}+2 m_{i}+\frac{1}{2} m_{i+1}=\beta_{i}, \quad i=1,2, \ldots, N-1
\end{array} .\right.
$$

From Eq. (16) we can get the following relation:

$$
\mathbf{A M}=\mathbf{E},
$$

where

$$
\mathbf{A}=\left[\begin{array}{ccccccccc}
1 & 0 & 0 & 0 & \cdots & 0 & 0 & 0 & 0 \\
0.5 & 2 & 0.5 & 0 & \cdots & 0 & 0 & 0 & 0 \\
0 & 0.5 & 2 & 0.5 & \cdots & 0 & 0 & 0 & 0 \\
0 & 0 & 0.5 & 2 & \cdots & 0 & 0 & 0 & 0 \\
\cdots & \cdots & \cdots & \cdots & \cdots & \cdots & \cdots & \cdots & \cdots \\
0 & 0 & 0 & 0 & \cdots & 2 & 0.5 & 0 & 0 \\
0 & 0 & 0 & 0 & \cdots & 0.5 & 2 & 0.5 & 0 \\
0 & 0 & 0 & 0 & \cdots & 0 & 0.5 & 2 & 0.5 \\
0 & 0 & 0 & 0 & \cdots & 0 & 0 & 0 & 1
\end{array}\right]
$$

$$
\mathbf{M}=\left[\begin{array}{l}
m_{0} \\
m_{1} \\
m_{2} \\
\vdots \\
m_{N-2} \\
m_{N-1} \\
m_{N}
\end{array}\right] \quad \mathbf{E}=\frac{3}{2 h}\left[\begin{array}{c}
2 h / 3 \\
e_{2}-e_{0} \\
e_{3}-e_{1} \\
\vdots \\
e_{N-1}-e_{N-3} \\
e_{N}-e_{N-2} \\
2 h / 3
\end{array}\right]
$$

Assuming that $\mathbf{B}=(3 / 2 h) \mathbf{A}^{-1}$, the interpolation weight of the $k$ th independent point at the $j$ th time point $\left(t_{j} \in\left[t_{i}, t_{i+1}\right]\right)$ can be expressed as

$$
\phi_{j, k}= \begin{cases}\left(\frac{j-t_{i+1}}{h}\right)^{2}\left(1+2 \frac{j-t_{i}}{h}\right)+\varphi_{j, k}, & (k=i) \\ \varphi_{j, k}, & (k \neq i, k \neq i+1), \\ \left(\frac{j-t_{i}}{h}\right)^{2}\left(1-2 \frac{j-t_{i+1}}{h}\right)+\varphi_{j, k}, & (k=i+1)\end{cases}
$$

where

$$
\varphi_{j, k}= \begin{cases}-B_{i, k+2}\left(\frac{j-t_{i+1}}{h}\right)^{2}\left(j-t_{i}\right)-B_{i+1, k+2}\left(\frac{j-t_{i}}{h}\right)^{2}\left(j-t_{i+1}\right), & (k=0,1) \\ \left(B_{i, k}-B_{i, k+2}\right)\left(\frac{j-t_{i+1}}{h}\right)^{2}\left(j-t_{i}\right)+\left(B_{i+1, k}-B_{i+1, k+2}\right)\left(\frac{j-t_{i}}{h}\right)^{2}\left(j-t_{i+1}\right), & (k=2,3, \ldots, N-2) . \\ B_{i, k}\left(\frac{j-t_{i+1}}{h}\right)^{2}\left(j-t_{i}\right)+B_{i+1, k}\left(\frac{j-t_{i}}{h}\right)^{2}\left(j-t_{i+1}\right), & (k=N-1, N)\end{cases}
$$




\section{Numerical experiments}

\section{a. Model settings}

Numerical experiments are performed to test the feasibility and reliability of the model and the interpolation methods in inversion of pollutant emission rate. The computation area is the Bohai Sea as shown in Fig. 1 $\left(37^{\circ}-41^{\circ} \mathrm{N}, 117.5^{\circ}-122.5^{\circ} \mathrm{E}\right)$ including Liaodong Bay, Bohai Bay, Laizhou Bay, and the central area. In this work, real bathymetry is employed. The horizontal resolution is $4^{\prime} \times 4^{\prime}$, and there are six layers in the vertical direction. The open boundary is set at $122.5^{\circ} \mathrm{E}$, and the $A_{H}$ and $K_{V}$ are set as $100 \mathrm{~m}^{2} \mathrm{~s}^{-1}$ and $10^{-5} \mathrm{~m}^{2} \mathrm{~s}^{-1}$, respectively. The time step interval is set to be $1 \mathrm{~h}$ and the total simulation time is $168 \mathrm{~h}$. The hydrodynamic background field is calculated by the Finite Volume Coastal Ocean Model (FVCOM; Chen et al. 2003). Many previous works have used this model for the study of tide and storm surge in the Bohai Sea (Zhao and Jiang 2011; Ding and Wei 2014). A nonoverlapping triangular mesh is configured in the Bohai Sea, and the horizontal resolution ranges from 0.4 to $0.8 \mathrm{~km}$. According to the description about the criteria for horizontal resolution and time step in Chen et al. (2003), the external and internal time steps are set as 20 and 120 s, respectively. The data are outputted from the model every hour. The spherical and $\sigma$ coordinates are set in the computational area, and there are six $\sigma$ layers in the vertical direction. According to $\mathrm{Lu}$ and Zhang (2006), the second-order bottom friction coefficient is set as $1.5 \times 10^{-3}$. Tidal boundary conditions, including the $\mathrm{M}_{2}$ constituent, are implemented in this model. The Blumberg and Kantha implicit radiation conditions are set as the temperature and salinity open boundary conditions (Chen et al. 2003).

\section{b. Design of numerical experiments}

One pollutant point source under the water is assumed in the study area. Then, 20 monitoring points are set around the point source (Fig. 1), and the observation scheme is planned as follows: points 1-4 are observed once per day on the second, the third, and the fifth days; points 5-12 are observed once per day on the fourth, the fifth, and the seventh days, and points 13-20 are observed once per day on the sixth and the seventh days. Details are listed in Table 1. Considering the variations of the pollutant emission rate, four types of time-varying emission rate are prescribed as follows:

type 1:

$$
\theta(t)=50+10 \times \cos (\pi \times t / 350),
$$

type 2:

$$
\theta(t)=50+10 \times \sin (\pi \times t / 350),
$$

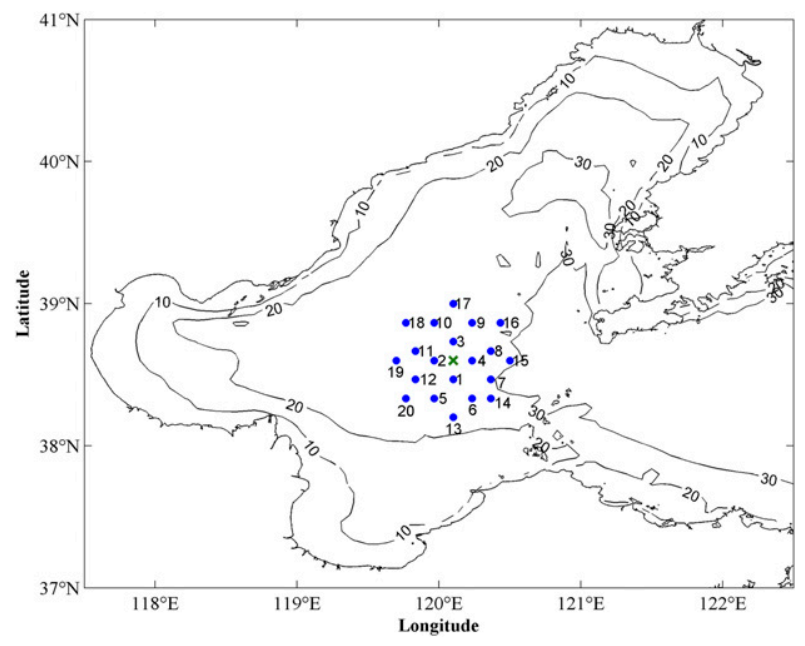

FIG. 1. The topography of the Bohai Sea (depth in meters), pollutant source (marked as " $\times$ "), and 20 monitoring stations (blue points).

type 3:

$$
\theta(t)=50+10 \times \sin (\pi \times t / 160),
$$

type 4:

$$
\theta(t)=50+10 \times \sin (\pi \times t / 80), \quad \text { where } \quad 1 \leq t \leq 168 .
$$

In the above four formulas, $t$ is in hours, the constant 50 is the mean pollutant emission rate, 10 is the varying amplitude of pollutant emission rate, and the constants $(80,160$, and 350) are the varying periods. All constants are prescribed based on the principle that the $\theta(t)$ changes in a physically reasonable range. The maximum is $60 \mathrm{mg} \mathrm{L}^{-1} \mathrm{~h}^{-1}$ and the minimum is $40 \mathrm{mg} \mathrm{L}^{-1} \mathrm{~h}^{-1}$, which guarantees the emission rate changes in a logical range and successful implementation of numerical experiments.

The entire computation process of the numerical experiments can be described as follows.

1) The "observations" are obtained through running the forward model [Eq. (1)] with one of these prescribed time-varying emission rates and the simulated pollutant concentration values at monitoring points are treated as observations.

2) We consider a constant pollution emission rate as an initial guess value at the independent time points.

3) The values at the independent time points are interpolated in order to reproduce the time series of the emission rate.

4) The pollutant concentration values at the monitoring points are simulated through the forward model [Eq. (1)] and the cost function computed [Eq. (3)]. 
TABLE 1. The observation scheme for TE1, TE2, TE3, and TE4.

\begin{tabular}{|c|c|c|c|c|c|c|c|c|}
\hline \multirow[b]{2}{*}{ Monitoring point } & \multirow[b]{2}{*}{ Longitude $\left({ }^{\circ}\right)$} & \multirow[b]{2}{*}{ Latitude $\left(^{\circ}\right)$} & \multicolumn{6}{|c|}{ Observation scheme } \\
\hline & & & Second & Third & Fourth & Fifth & Sixth & Seventh \\
\hline 1 & 120.1000 & 38.4667 & $\sqrt{ }$ & $\sqrt{ }$ & & $\sqrt{ }$ & & \\
\hline 2 & 119.9667 & 38.6000 & $\sqrt{ }$ & $\sqrt{ }$ & & $\sqrt{ }$ & & \\
\hline 3 & 120.1000 & 38.7333 & $\sqrt{ }$ & $\sqrt{ }$ & & $\sqrt{ }$ & & \\
\hline 4 & 120.2333 & 38.6000 & $\sqrt{ }$ & $\sqrt{ }$ & & $\sqrt{ }$ & & \\
\hline 5 & 119.9667 & 38.3333 & & & $\sqrt{ }$ & $\sqrt{ }$ & & $\sqrt{ }$ \\
\hline 6 & 120.2333 & 38.3333 & & & $\sqrt{ }$ & $\sqrt{ }$ & & $\sqrt{ }$ \\
\hline 7 & 120.3667 & 38.4667 & & & $\sqrt{ }$ & $\sqrt{ }$ & & $\sqrt{ }$ \\
\hline 8 & 120.3667 & 38.6667 & & & $\sqrt{ }$ & $\sqrt{ }$ & & $\sqrt{ }$ \\
\hline 9 & 120.2333 & 38.8667 & & & $\sqrt{ }$ & $\sqrt{ }$ & & $\sqrt{ }$ \\
\hline 10 & 119.9667 & 38.8667 & & & $\sqrt{ }$ & $\sqrt{ }$ & & $\sqrt{ }$ \\
\hline 11 & 119.8333 & 38.6667 & & & $\sqrt{ }$ & $\sqrt{ }$ & & $\sqrt{ }$ \\
\hline 12 & 119.8333 & 38.4667 & & & $\sqrt{ }$ & $\sqrt{ }$ & & $\sqrt{ }$ \\
\hline 13 & 120.1000 & 38.2000 & & & & & $\sqrt{ }$ & $\sqrt{ }$ \\
\hline 14 & 120.3667 & 38.3333 & & & & & $\sqrt{ }$ & $\sqrt{ }$ \\
\hline 15 & 120.5000 & 38.6000 & & & & & $\sqrt{ }$ & $\sqrt{ }$ \\
\hline 16 & 120.4333 & 38.8667 & & & & & $\sqrt{ }$ & $\sqrt{ }$ \\
\hline 17 & 120.1000 & 39 & & & & & $\sqrt{ }$ & $\sqrt{ }$ \\
\hline 18 & 119.7667 & 38.8667 & & & & & $\sqrt{ }$ & $\sqrt{ }$ \\
\hline 19 & 119.7000 & 38.6000 & & & & & $\sqrt{ }$ & $\sqrt{ }$ \\
\hline 20 & 119.7667 & 38.3333 & & & & & $\sqrt{ }$ & $\sqrt{ }$ \\
\hline
\end{tabular}

5) The initial guess values at the independent time points are adjusted through the adjoint method.

6) Calculation stops if the termination condition is reached; otherwise, go back to step 3 .

Finally, the most appropriate time-varying pollutant emission rate is obtained through above procedure. The ideal experiment based on the emission rates of type 1 is named TE1. Similarly, TE2, TE3, and TE4 are implemented based on the emission rates of type 2 , type 3 , and type 4 , respectively.

The "observations" in TE1-TE4 are obtained through strict mathematical calculation, meaning that there is no error in these so-called observations. However, the situ observations definitely contain noise. Therefore, a set of error experiments are implemented for contrasting the performance of SI and CI. Thus, observations containing artificial errors are applied into those error experiments. These artificial errors are produced by the random number generator. As a case study, those experiments are conducted based on TE4, of which the maximum percentage errors are 5\% (TE4-1), 10\% (TE4-2), 15\% (TE4-3), and 20\% (TE4-4). Everything else is the same except for the observations for TE4, TE4-1, TE4-2, TE4-3, and TE4-4.

Except for the interpolation method and error, the number of assimilated observations also may influence the accuracy of inversion results. For this, a series of experiments are carried out for comparison, with different schemes and numbers of observation. As a case study, these comparison experiments are based on TE3 and named TE3-1, TE3-2, TE3-3, and TE3-4.

For the experiments TE3-1 and TE3-2, the observation schemes are set as once and twice per day for all 20 monitoring points on the second to the seventh day. For the experiment TE3-3, the observation scheme is set as follows: points $1-4$ are observed three times a day (the interval is $8 \mathrm{~h}$ ) on the second, the third, and the fifth days; points $5-12$ are observed twice a day (the interval is $12 \mathrm{~h}$ ) on the fourth, the fifth, and the seventh days; and points 13-20 are observed once per day on the fourth, the sixth, and the seventh days. The observation scheme of TE3-4 is planned as follows: points 1-12 are observed three times a day (the interval is $8 \mathrm{~h}$ ) on the second, the third, and the fifth days; and points 13-20 are observed twice a day (the interval is $12 \mathrm{~h}$ ) on the fourth, the sixth, and the seventh days. The total numbers of observations are 120,240,108, and 156 for TE3-1, TE3-2, TE3-3, and TE3-4, respectively.

The selection of the independent time points should depend on the specific situation. First, the purpose of

TABLE 2. MAEs $\left(\mathrm{mg} \mathrm{L}^{-1} \mathrm{~h}^{-1}\right)$ between the prescribed and inverted emission rates by two interpolation methods for TE1, TE2, TE3, and TE4 (M0 is the MAE calculated using the initial guess values, and M100 indicates that the MAE is obtained through 100 iterations).

\begin{tabular}{|c|c|c|c|c|c|c|c|c|}
\hline \multirow{2}{*}{$\begin{array}{l}\text { Interpolation } \\
\text { method }\end{array}$} & \multicolumn{2}{|c|}{ TE1 } & \multicolumn{2}{|c|}{ TE2 } & \multicolumn{2}{|c|}{ TE3 } & \multicolumn{2}{|c|}{ TE4 } \\
\hline & M0 & M100 & M0 & M100 & M0 & M100 & M0 & M100 \\
\hline CI & & 0.14 & 58 & 0.35 & 73 & 0.55 & 22 & 1.33 \\
\hline SI & & 0.05 & & 0.14 & & 0.49 & & 0.37 \\
\hline
\end{tabular}


TABLE 3. RMSEs $\left(\mathrm{mg} \mathrm{L}^{-1} \mathrm{~h}^{-1}\right)$ between the prescribed and inverted emission rates by two interpolation methods for TE1, TE2, TE3, and TE4 (R0 is the RMSE calculated using the initial guess values, and R100 indicates that the RMSE is obtained through 100 iterations).

\begin{tabular}{|c|c|c|c|c|c|c|c|c|}
\hline \multirow{2}{*}{$\begin{array}{l}\text { Interpolation } \\
\text { method }\end{array}$} & \multicolumn{2}{|c|}{ TE1 } & \multicolumn{2}{|c|}{ TE2 } & \multicolumn{2}{|c|}{ TE3 } & \multicolumn{2}{|c|}{ TE4 } \\
\hline & $\mathrm{R} 0$ & $\mathrm{R} 100$ & $\mathrm{R} 0$ & R100 & R0 & R100 & $\mathrm{R} 0$ & $\mathrm{R} 1$ \\
\hline $\begin{array}{l}\text { CI } \\
\text { SI }\end{array}$ & 3.54 & $\begin{array}{l}0.29 \\
0.08\end{array}$ & 5.26 & $\begin{array}{l}0.38 \\
0.17\end{array}$ & 3.92 & $\begin{array}{l}0.87 \\
0.80\end{array}$ & 12.99 & \\
\hline
\end{tabular}

this paper is to compare the performance of different interpolation methods in estimation of pollutant emission rate. Therefore, these control experiments should be the same except for interpolation method. So, the selection of independent time points can be determined according to the fourth prescribed emission rate [Eq. (25)], because it is the most complicated among the four prescribed emission rates. Second, the original purpose of applying the independent points is to reduce the quantity of variables to be inverted and to promote the efficiency of the adjoint assimilation model. Therefore, the number of independent points should be as few as possible under the premise that the inversion is accurate. Based on the above-mentioned two principles, seven independent time points are selected after lots of trial. They are set at the 1st, 29th, 57th, 85th, 113th, 141st, and 168th hour, respectively. The same independent points are used in all numerical experiments of this paper.

\section{c. Experimental results}

The hydrodynamic background data calculated by FVCOM are processed with harmonic analysis. The cotidal chart is available in Liu et al. (2017), and comparison with many previous works (Zhao and Jiang 2011; Yao et al. 2012; Ding and Wei 2014) demonstrates the reliability of this hydrodynamic background field. Then, the field is transformed into $z$ coordinates and interpolated into the $4^{\prime} \times 4^{\prime}$ square grid using the kriging interpolation method (Krige 1952). Such pretreatment is implemented in all above numerical experiments.

The cost function, normalized by the value at the first iteration step, is a significant criterion for evaluating the

TABLE 4. MAEs $\left(\mathrm{mg} \mathrm{L}^{-1} \mathrm{~h}^{-1}\right)$ between the given and inverted emission rates by two interpolation methods for TE4, TE4-1, TE4-2, TE4-3, and TE4-4.

\begin{tabular}{cccccc}
\hline \hline & \multicolumn{5}{c}{ MAEs } \\
\cline { 2 - 6 } $\begin{array}{c}\text { Interpolation } \\
\text { method }\end{array}$ & $\begin{array}{c}\text { TE4 } \\
(0)\end{array}$ & $\begin{array}{c}\text { TE4-1 } \\
(5 \%)\end{array}$ & $\begin{array}{c}\text { TE4-2 } \\
(10 \%)\end{array}$ & $\begin{array}{c}\text { TE4-3 } \\
(15 \%)\end{array}$ & $\begin{array}{c}\text { TE4-4 } \\
(20 \%)\end{array}$ \\
\hline CI & 1.33 & 2.05 & 2.26 & 2.44 & 2.62 \\
SI & 0.37 & 1.37 & 1.77 & 2.02 & 2.23 \\
\hline
\end{tabular}

TABLE 5. RMSEs $\left(\mathrm{mg} \mathrm{L}^{-1} \mathrm{~h}^{-1}\right)$ between the given and inverted emission rates by two interpolation methods for TE4, TE4-1, TE4-2, TE4-3, and TE4-4.

\begin{tabular}{|c|c|c|c|c|c|}
\hline \multirow[b]{2}{*}{$\begin{array}{c}\text { Interpolation } \\
\text { method }\end{array}$} & \multicolumn{5}{|c|}{ RMSEs } \\
\hline & $\begin{array}{l}\text { TE4 } \\
(0)\end{array}$ & $\begin{array}{c}\text { TE4-1 } \\
(5 \%)\end{array}$ & $\begin{array}{l}\text { TE4-2 } \\
(10 \%)\end{array}$ & $\begin{array}{l}\text { TE4-3 } \\
(15 \%)\end{array}$ & $\begin{array}{l}\text { TE4-4 } \\
(20 \%)\end{array}$ \\
\hline CI & 1.59 & 2.42 & 2.75 & 3.07 & 3.33 \\
\hline SI & 0.49 & 1.67 & 2.22 & 2.68 & 3.10 \\
\hline
\end{tabular}

inversion process. The mean absolute error (MAE) and the root-mean-square error (RMSE) are applied to evaluate the inversion results. The MAE is the mean value of the sum of absolute error between inverted and prescribed pollutant emission rate, which can be calculated through the following equation:

$$
\text { MAE }=\frac{\sum_{i=1}^{N}\left|I_{i}-P_{i}\right|}{N},
$$

where $I$ is the inverted pollutant emission rate, $P$ is the prescribed pollutant emission rate, and $N$ is the number of time points. In this paper, the number of 120 is selected as time series, which is an effective length (the reason is made clear in the appendix). Therefore, the figures (Figs. 3-5) are shown from 1 to $120 \mathrm{~h}$, and the results (see Tables 2-7) are calculated with $120 \mathrm{~h}$.

The normalized cost functions (NCFs) of TE1-TE4 are shown in Fig. 2, which reveals the changes of NCFs with iterations for the four prescribed types of emission rate. It can be deduced that the four given types of emission rate are inverted successfully because all NCFs are below $1 \times 10^{-3}$ after 100 iterations.

Tables 2 and 3 list the MAEs and RMSEs, respectively, giving a more powerful indicator for comparison of the performances of SI and CI in inverting the timevarying pollutant emission rate. In Table $2, \mathrm{M} 0$ means the MAE calculated using the initial guess values at the independent time points, and M100 represents the MAE calculated using the adjusted initial guess values at the independent time points after 100 iterations. The same is true for R0 and R100 in Table 3. For the four given types of emission rate, MAEs and RMSEs are

TABLE 6. MAEs $\left(\mathrm{mg} \mathrm{L}^{-1} \mathrm{~h}^{-1}\right)$ between the given and inverted emission rate by two interpolation methods for TE3, TE3-1, TE3-2, TE3-3, and TE3-4.

\begin{tabular}{cccccc}
\hline \hline $\begin{array}{c}\text { Interpolation } \\
\text { method }\end{array}$ & TE3 & TE3-1 & TE3-2 & TE3-3 & TE3-4 \\
\cline { 2 - 6 } & 0.548 & 0.365 & 0.357 & 0.544 & 0.538 \\
CI & 0.491 & 0.290 & 0.312 & 0.484 & 0.484 \\
SI & & & &
\end{tabular}


TABLE 7. RMSEs $\left(\mathrm{mg} \mathrm{L}^{-1} \mathrm{~h}^{-1}\right)$ between the given and inverted emission rate by two interpolation methods for TE3, TE3-1, TE3-2, TE3-3, and TE3-4.

\begin{tabular}{|c|c|c|c|c|c|}
\hline \multirow{2}{*}{$\begin{array}{c}\text { Interpolation } \\
\text { method }\end{array}$} & \multicolumn{5}{|c|}{ RMSEs } \\
\hline & TE3 & TE3-1 & TE3-2 & TE3-3 & TE3-4 \\
\hline $\mathrm{CI}$ & 0.870 & 0.442 & 0.423 & 0.863 & 0.858 \\
\hline SI & 0.804 & 0.372 & 0.419 & 0.808 & 0.812 \\
\hline
\end{tabular}

reduced significantly with iterations. For the MAE, the least improvement after 100 iterations is $79.85 \%$ for the $\mathrm{CI}$ and $82.05 \%$ for the SI in the TE3, and more than $88 \%$ for all others. It is similar to the RMSE that the least improvement after 100 iterations is $77.81 \%$ for the CI and $79.59 \%$ for the SI in TE3. Those improvements prove the validity of the adjoint method. More importantly, the MAEs and RMSEs of the SI are all less than those of the CI, showing the improvements by choosing the SI. The least improvements are $10.91 \%$ for the MAE and $8.05 \%$ for the RMSE in TE3, and the most improvements are $72.18 \%$ for the MAE in TE4 and $72.41 \%$ for the RMSE in TE1. Those improvements indicate the advantage of the SI over the CI in the accuracy of the inversion result.

The inversion results of the four prescribed types of emission rate are illustrated in Fig. 3. It can be found that inversion results with both interpolation methods reflect the basic structure features of the four prescribed types of emission rate. However, the black solid lines representing the inversion results obtained by the SI are closer to the prescribed values. This is consistent with the conclusion from Tables 2 and 3. Furthermore, it is worthwhile to note that the black solid lines are as smooth as the prescribed curves, but the red solid lines representing the inversion results obtained by the CI are fairly rough. So, the advantage of the SI in expressing the smoothness is revealed.
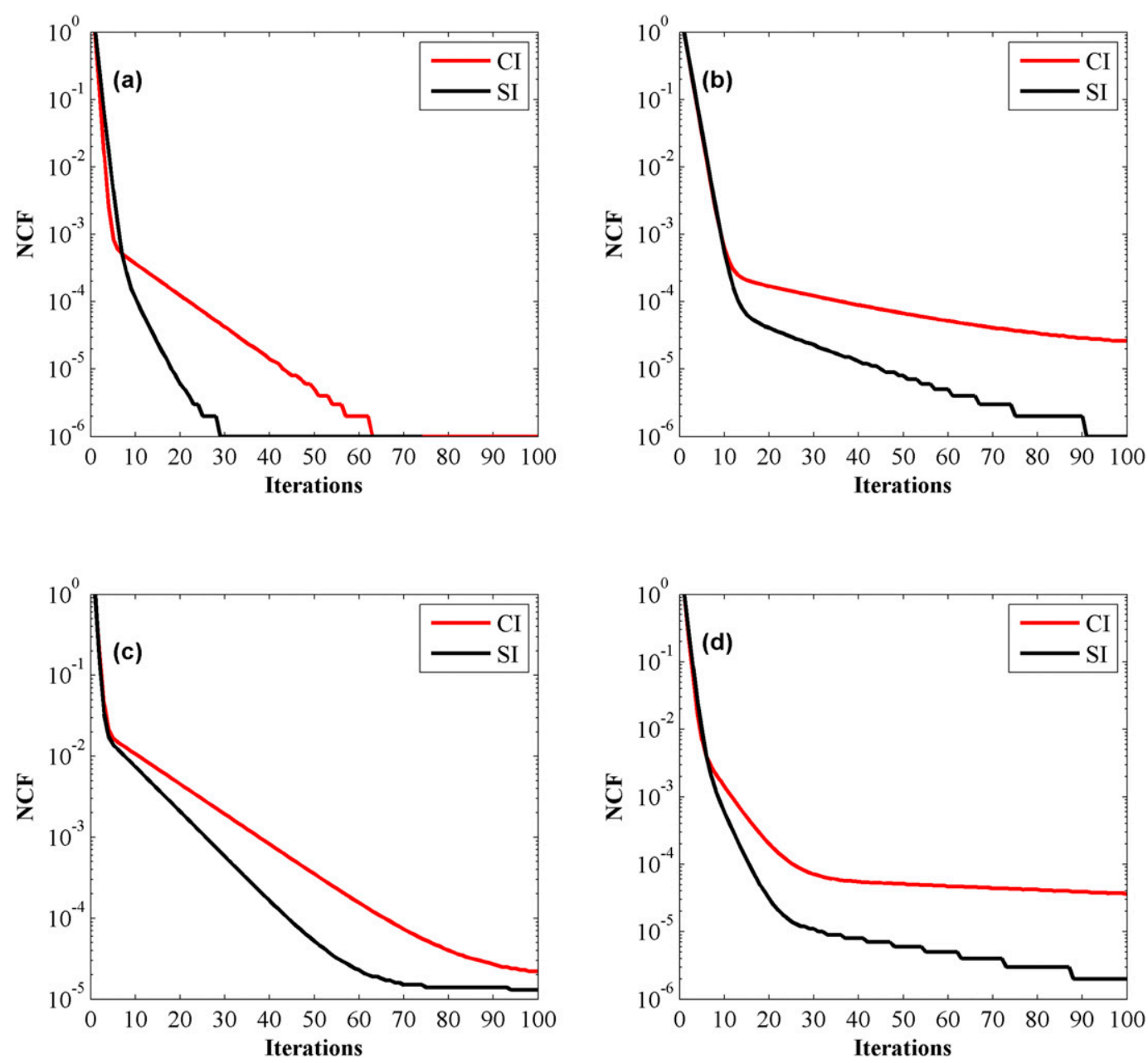

FIG. 2. Normalized cost functions (NCFs) of (a) TE1, (b) TE2, (c) TE3, and (d) TE4. Note that, in all panels, the red solid lines are the NCFs by the Cressman interpolation (CI) and the black solid lines are those by the spline interpolation (SI). 

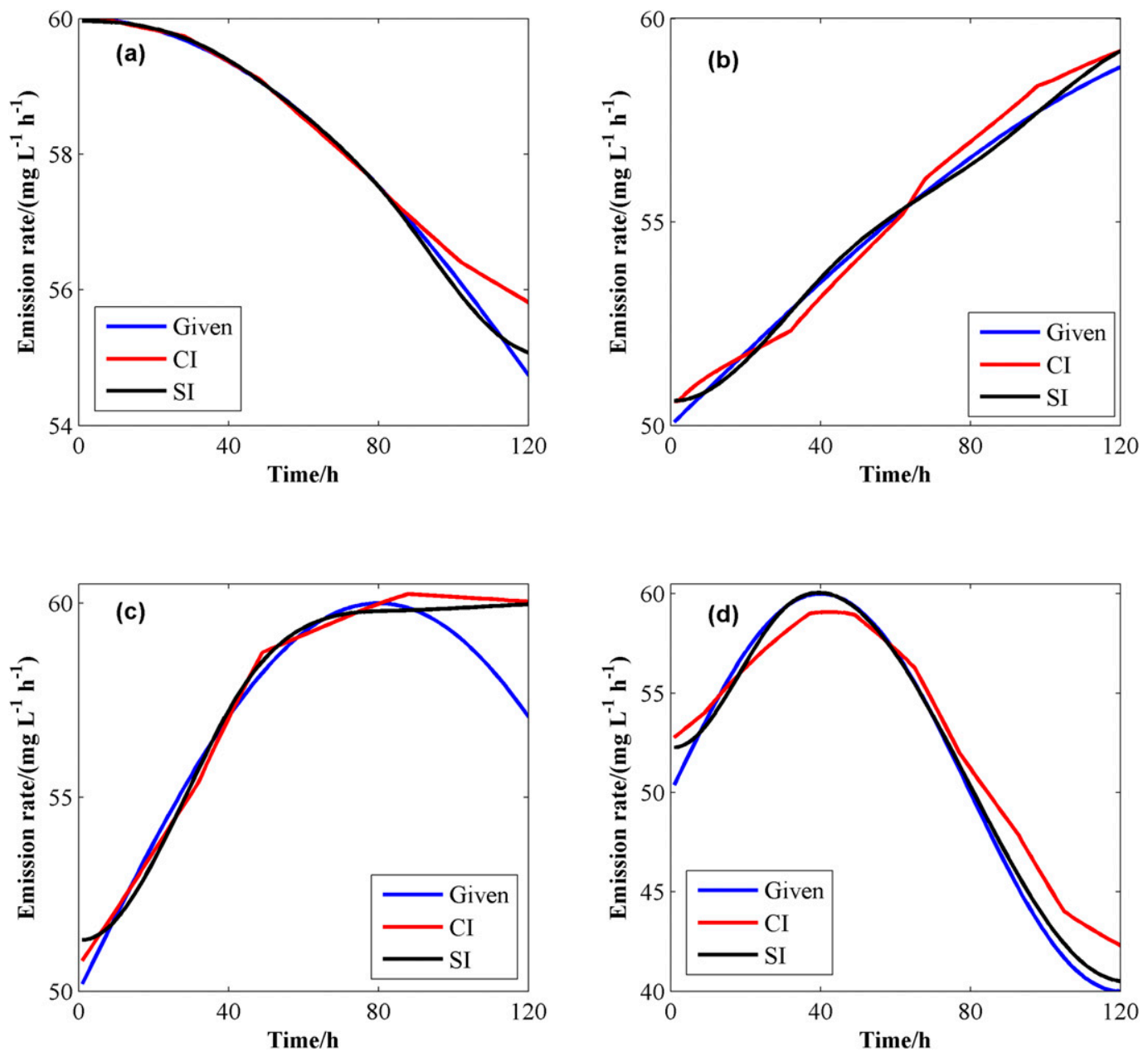

FIG. 3. The prescribed and inverted pollutant emission rates of (a) TE1, (b) TE2, (c) TE3, and (d) TE4. Note that, in all panels, the blue solid lines are the prescribed emission rates, and the red solid lines and the black solid lines are the inverted emission rates by the CI and SI, respectively.

The effect of error on the inversion results is shown in Fig. 4, which is obvious through the comparison with the inversion in TE4. The error in assimilated "observations" increases the deviation between the prescribed emission rates and inversion results based on TE4, and the larger the error is, the larger the deviation is. But, it still can be seen that the inversion results obtained by the SI are smooth curves.

Tables 4 and 5 list, respectively, the MAEs and RMSEs of TE4-1 through TE4-4 under the effect of error. Along with the increasing error, the MAEs and RMSEs are increased for both interpolation methods. Also, the improvement of MAE for the inversion results obtained by SI is reduced continuously, from $72.18 \%$ for the error-free experiment (TE4) to $31.71 \%$ (TE4-1), $18.70 \%$ (TE4-2), $17.21 \%$ (TE4-3), and 14.89\% (TE4-4). For the RMSEs, the improvement by choosing the SI is reduced from $69.18 \%$ (TE4) to $30.99 \%$ (TE4-1), $19.27 \%$
(TE4-2), 12.70\% (TE4-3), and 6.91\% (TE4-4). The error weakens the improvement by applying the SI. However, under the same error, the MAEs and RMSEs obtained by SI are less than those obtained by CI. Even the MAE of the "observations" containing higher error obtained by SI is less than that containing lower error obtained by CI. For example, the MAE obtained by SI in TE4-2 $(10 \%)$ is less than that obtained by CI in TE4-1 $(5 \%)$. The MAE obtained by SI in TE4-3 (15\%) is less than that obtained by CI in TE4-1 (5\%) and TE4-2 (10\%). A similar conclusion can be found from the RMSEs, which shows that the advantage of SI in the accuracy of inversion results is still valid even under the effect of error.

Figure 5 exhibits the inversion results of experiments with different numbers of observations to be assimilated, and the MAEs and RMSEs are shown in Tables 6 and 7 , respectively. Those comparison experiments are based on TE3, so the inversion results of TE3 (Fig. 5a) 

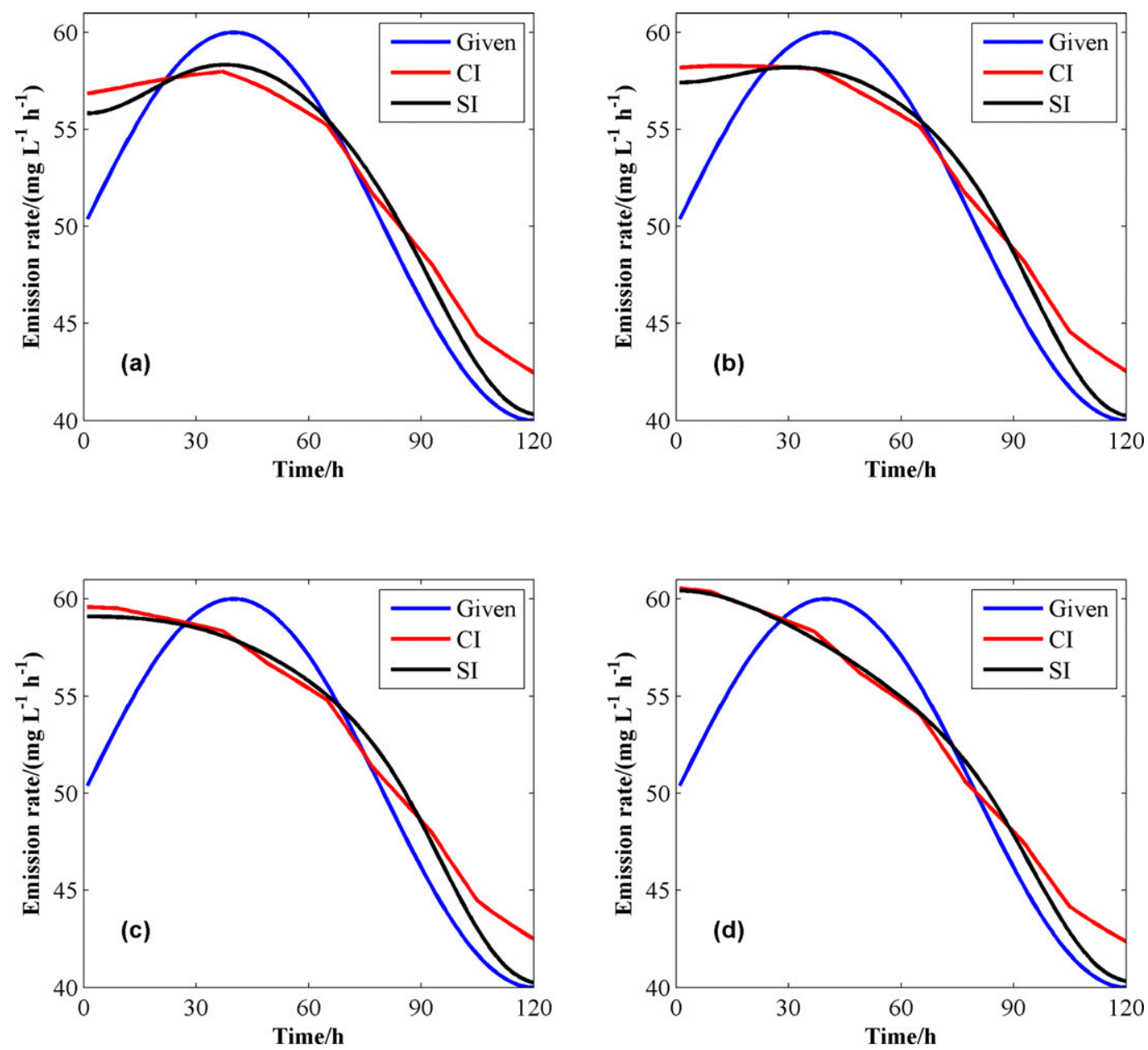

FIG. 4. The prescribed and inverted pollutant emission rates of (a) TE4-1, (b) TE4-2, (c) TE4-3, and (d) TE4-4. Note that, in all panels, the blue solid lines are the prescribed emission rates, and the red solid lines and the black solid lines are the inverted emission rates by the CI and SI, respectively.

are also displayed for the comparison. Figures $5 \mathrm{~b}-\mathrm{e}$ are the inversion results of experiments TE3-1, TE3-2, TE3-3, and TE3-4, respectively, which assimilate 120, 240, 108, and 156 observations respectively. For better comparison of inversion results with different numbers of observations, three significant digits are kept in Tables 6 and 7.

From Fig. 5, it is obvious that the inversion results in Figs. $5 \mathrm{~b}$ and $5 \mathrm{c}$ are the closest to the prescribed pollutant emission rate. For the two subfigures, it seems that the inversion results in Fig. $5 \mathrm{c}$ are better, which reflects the downswing of emission rate from 90 to $120 \mathrm{~h}$ well. However, there is almost no difference among the others subfigures in Fig. 5. It can be verified from Tables 6 and 7 that the MAEs and the RMSEs of TE3-1 and TE3-2 are the least among the five experiments, and the others are very close. As a result, the effect of observation number is discerned to be that the inversion result is more similar to the prescribed emission rate with more observations for both interpolation methods. In addition, with the smoother inverted emission rates (Fig. 5), the lower MAEs and RMSEs (Tables 6 and 7) obtained by the SI again demonstrate the advantage of SI in smoothness and accuracy.

Through the aforementioned numerical experiments, satisfactory results can be obtained by both interpolation methods. However, there are still significant improvements in accuracy and smoothness by using the SI.

\section{Discussion}

The above numerical experiments demonstrate the application of SI results in a successful and better inversion of the pollutant emission rate, in terms of the smoothness and accuracy of the inversion results. The priority of SI in smoothness and accuracy had been 

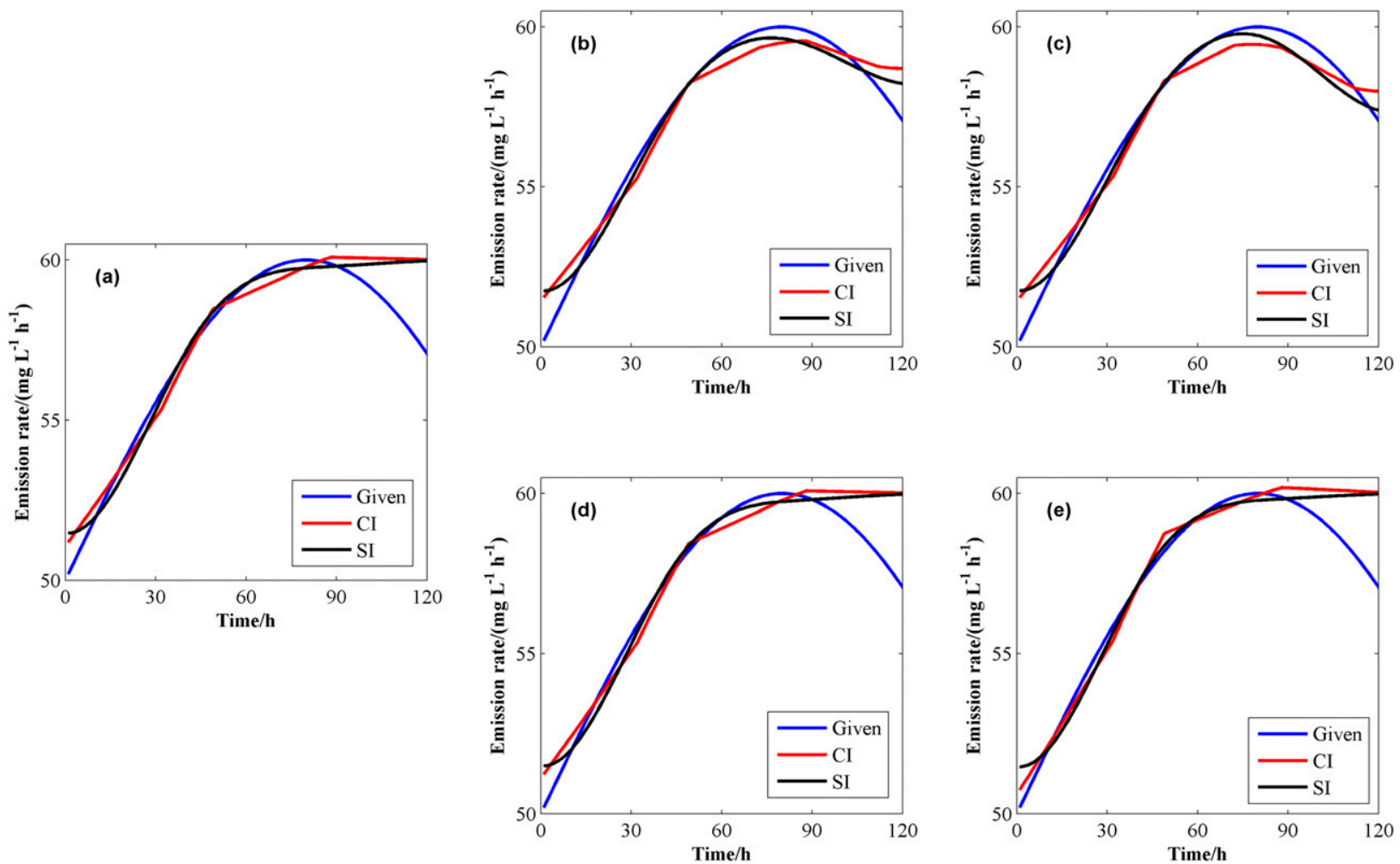

FIG. 5. The prescribed and inverted pollutant emission rates of (a) TE3, (b) TE3-1, (c) TE3-2, (d) TE3-3, and (e) TE3-4. Note that, in all panels, the blue solid lines are the prescribed emission rates, and the red solid lines and the black solid lines are the inverted emission rates by the $\mathrm{CI}$ and SI, respectively.

revealed in many previous works (Perrin et al. 1987; Unser et al. 1991; Wijnands et al. 2016; Pan et al. 2017), so it is a natural characteristic for the SI. It is appropriate and necessary for the SI to be introduced into the estimation of pollutant emission rates.

Error is inevitable in situ observations, so the attention should be paid to searching for ways to weaken the effects of observation error. Through the comparison of error experiments (TE4-1, TE4-2, TE4-3, and TE4-4), it is demonstrated that the SI could reduce the MAE more than the CI. So, the SI method is more applicable for the situ observations. Furthermore, these error experiments also exhibit the level of MAE when the SI is applied to situ observations containing errors. Comparing MAEs obtained by SI in TE4-1 and TE4- 2 with that obtained by CI in TE4, it can be found that when the SI is applied to situ observations containing errors, the MAE is at the same level with that obtained by CI for observations that are ideally error free. At this point, the effect of error on inversion results could be ignored completely when SI is applied.

The observation scheme is necessary before carrying out the situ observation. A good scheme means the cost is low but the data amount is sufficient. Through comparing the inversion results of TE3, TE3-1, TE3-2, TE3-3, and TE3-4, it can be deduced that adding more observations is useful for improving inversion results for both interpolation methods. However, an inconsistent phenomenon should be noticed regarding the changes of MAEs or RMSEs with the adding of numbers of observations by the CI and SI. The MAEs or RMSEs obtained by the CI decrease with more observations, while those obtained by the SI are invariant (TE3-3, TE3-4) or even increased (TE3-1, TE3-2). We do not know why the MAEs or RMSEs increase with more observations, but it is clear that there is a maximum of the number of observations for the SI. In other words, the number of observations is already sufficient for the SI; however, more observations are needed for the CI. Also, all MAEs and RMSEs obtained by the SI are less than those obtained by the CI. Therefore, fewer observations are needed for the SI to achieve the same accuracy, which means lower data amounts and observation costs.

\section{Conclusions}

In this paper, an ocean pollutant diffusion model with the adjoint method is used to reconstruct the time-varying 

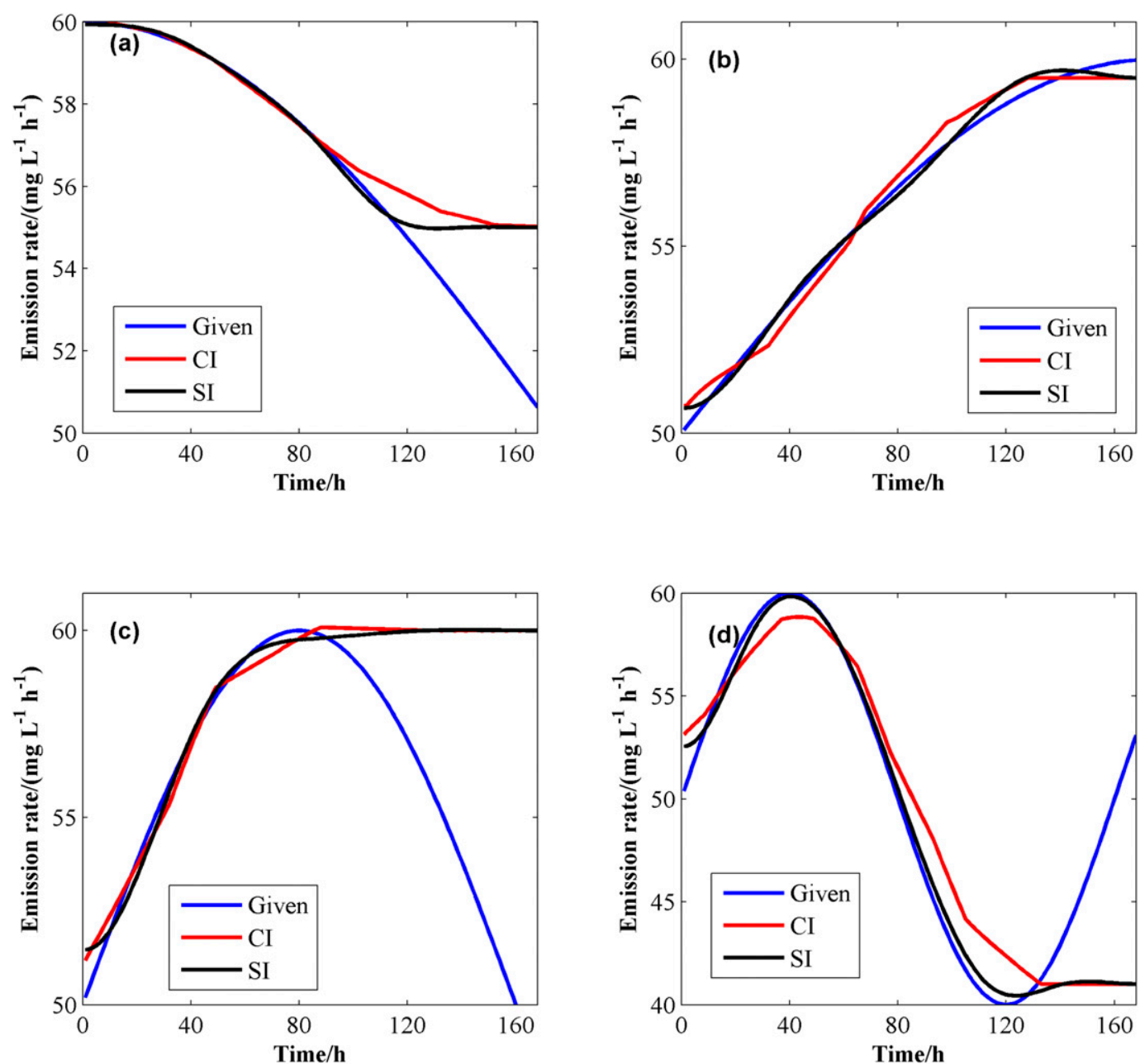

FIG. A1. The prescribed and inverted pollutant emission rates of (a) TE1, (b) TE2, (c) TE3, and (d) TE4. Note that, in all panels, the blue solid lines are the prescribed emission rates, and the red solid lines and the black solid lines are the inverted emission rates by the CI and SI, respectively.

emission rate of a known pollutant point source in ocean. To solve the ill-posedness of the inversion problem, IPS is implemented with different interpolation methods, the $\mathrm{CI}$ and the SI. The model with different interpolation methods is calibrated by a series of numerical experiments.

Experimental results show that four prescribed types of pollutant emission rate are inverted successfully by both interpolation methods, but the application of SI improves the inversion results in most cases. The improvement focuses on the smoothness and accuracy of the inversion results. In error experiments, compared with the CI, the SI has a better performance in resisting the influence of error. The accuracy of inversion results obtained by SI for the situ observations containing errors is at the same level with that obtained by $\mathrm{CI}$ for observations that are error free ideally. Furthermore, to achieve the same accuracy of inversion, fewer observations are needed for the SI than for the CI. For the SI, better resistance to the impact of error and demand for fewer observations give rise to better appropriateness in practice.

Acknowledgments. We appreciate financial support from the Key Research and Development Plan of Shandong Province through Grant 2016ZDJS09A02, the National Natural Science Foundation of China through Grant 41606006, the National Key Research and Development Plan of China through Grant 2016YFC1402304, and the Fundamental Research Funds for the Central Universities.

\section{APPENDIX}

\section{The Selection of Effective Length}

This part explains the reason why the inversion results in Fig. 3 stop at $120 \mathrm{~h}$ although the simulation time spans 

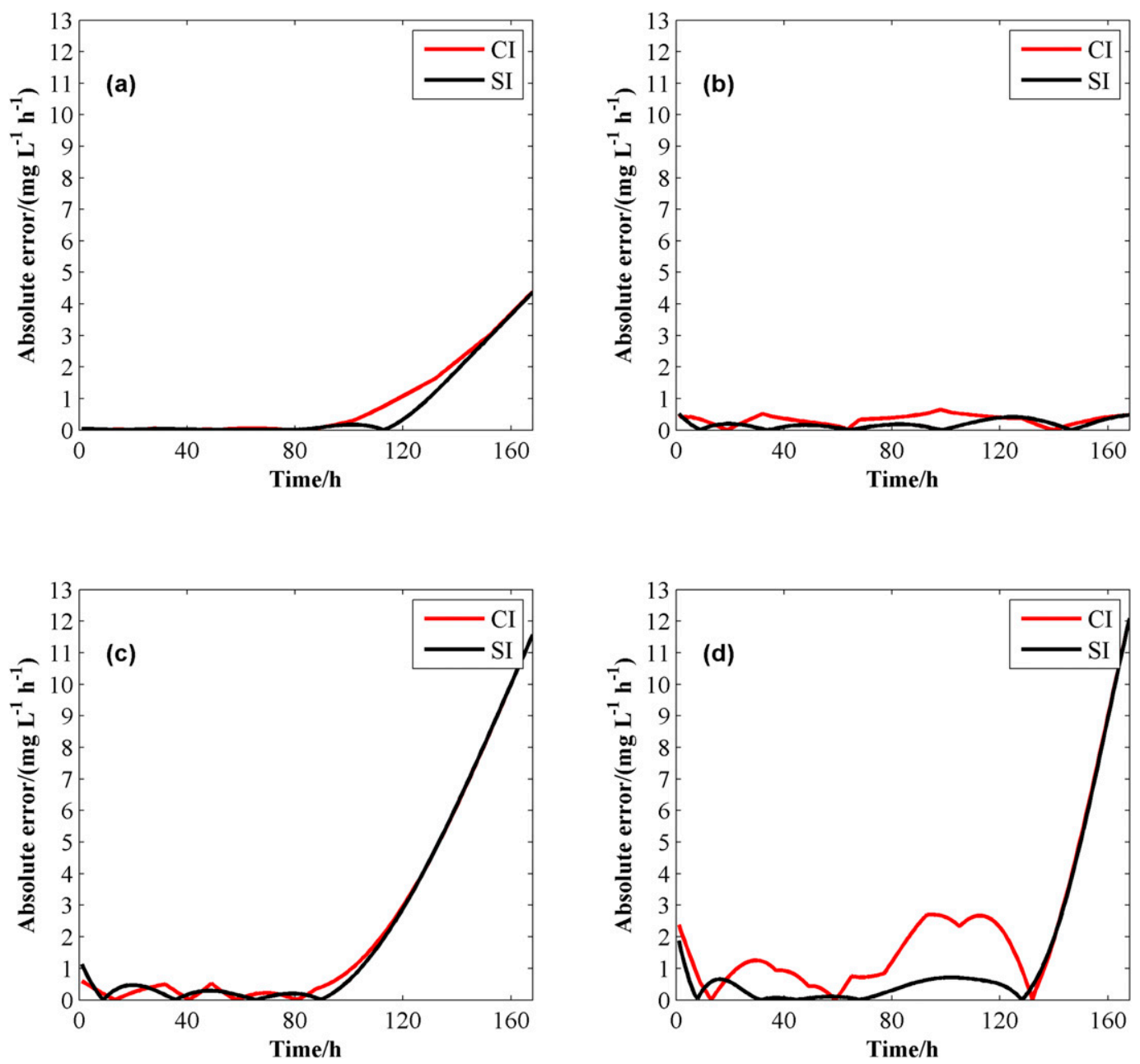

FIG. A2. The absolute errors between the inverted and prescribed pollutant emission rate for (a) TE1, (b) TE2, (c) TE3, and (d) TE4. Note that, in all panels, the red solid lines and the black solid lines are the inverted emission rates by the $\mathrm{CI}$ and SI, respectively.

to $168 \mathrm{~h}$. In fact, the pollutant emission rate during 120 to $168 \mathrm{~h}$ can also be inverted. However, the difference between the inverted and prescribed pollutant emission rate is large during this period (Fig. A1). This is caused by the limitation of the adjoint method. In the adjoint model, the calculation process is implemented in a reverse chronological order. So, the initial field of the adjoint model should be the distribution of the pollutant concentration at the 168th hour. However, this distribution cannot be given appropriately before the calculation of adjoint model. Therefore, it needs time to be optimized by adjusting the values at independent time points. This is the reason why the inversion results are bad during 120 to $168 \mathrm{~h}$.

There are two reasons for the selection of the time $120 \mathrm{~h}$ when the figures and tables stop. First, the absolute error between the inverted and prescribed pollutant emission rate is set as less than $3 \mathrm{mg} \mathrm{L}^{-1} \mathrm{~h}^{-1}$. Too much error will result in less reliability of the inversion results. Figure A2 shows the change of absolute error with the time. It can be found from Fig. A2c that the absolute error exceeds $3 \mathrm{mg} \mathrm{L}^{-1} \mathrm{~h}^{-1}$ when the time exceeds $120 \mathrm{~h}$. So, the time $120 \mathrm{~h}$ is selected. In addition, the selection of time $120 \mathrm{~h}$ considers the experiments TE3-1, TE3-2, TE3-3, and TE3-4. Because those experiments are conducted to test the influence of "observation" number on inversion result, it can be found that the deviation between the inverted and prescribed emission rate gets increasingly larger from 80 to $120 \mathrm{~h}$ in Fig. 3c. The objective for reserving this period is to test if the deviation gets smaller when the number of observations is increased. Thus, the selection of $120 \mathrm{~h}$ is appropriate.

Also, it is possible to invert the pollutant emission rate acceptably during 120 to $168 \mathrm{~h}$ by extending the total 
simulation time, adding more observations, and adjusting the number and locations of independent time points. But, it is not the key point and is beyond the scope of this paper.

\section{REFERENCES}

Atmadja, J., and A. C. Bagtzoglou, 2001: Pollution source identification in heterogeneous porous media. Water Resour., 37, 2113-2125, https://doi.org/10.1029/2001WR000223.

Barnes, S. L., 1964: A technique for maximizing details in numerical weather map analysis. J. Appl. Meteor., 3, 396-409, https://doi.org/ 10.1175/1520-0450(1964)003<0396:ATFMDI > 2.0.CO;2.

Chen, C. S., H. D. Liu, and R. C. Beardsley, 2003: An unstructured grid, finite-volume, three-dimensional, primitive equations ocean model: Application to coastal ocean and estuaries. J. Atmos. Oceanic Technol., 20, 159-186, https://doi.org/ 10.1175/1520-0426(2003)020<0159:AUGFVT>2.0.CO;2.

Chen, H., A. Cao, J. Zhang, C. Miao, and X. Lv, 2014: Estimation of spatially varying open boundary conditions for a numerical internal tidal model with adjoint method. Math. Comput. Simul., 97, 14-38, https://doi.org/10.1016/j.matcom.2013.08.005.

Chen, Z., K. An, Y. Liu, and W. Chen, 2014: Adjoint method for an inverse problem of CCPF model. Chin. Ann. Math., 35B, 337354, https://doi.org/10.1007/s11401-014-0837-9.

Correa, S. B., J. K. Araujo, J. M. F. Penha, C. N. Da Cunha, P. R. Stevenson, and J. T. Anderson, 2015: Overfishing disrupts an ancient mutualism between frugivorous fishes and plants in Neotropical wetlands. Biol. Conserv., 191, 159-167, https:// doi.org/10.1016/j.biocon.2015.06.019.

Cressman, G. W., 1959: An operational objective analysis system. Mon. Wea. Rev., 87, 367-374, https://doi.org/10.1175/ 1520-0493(1959)087<0367:AOOAS > 2.0.CO;2.

Datta, B., D. Chakrabarty, and A. Dhar, 2011: Identification of unknown groundwater pollution sources using classical optimization with linked simulation. J. Hydro-environ. Res., 5, 25 36, https://doi.org/10.1016/j.jher.2010.08.004.

Ding, Y., and H. Wei, 2014: Research of the influential factors on the simulation of storm surge in the Bohai Sea. Open Mech. Eng. J., 8, 151-156, https://doi.org/10.2174/1874155X20140501010.

Eyring, V., H. W. Köhler, J. Van Aardenne, and A. Lauer, 2005: Emissions from international shipping: 1 . The last 50 years. J. Geophys. Res., 110, D17305, https://doi.org/10.1029/ 2004JD005619.

Fan, W., and X. Lv, 2009: Data assimilation in a simple marine ecosystem model based on spatial biological parameterizations. Ecol. Modell., 220, 1997-2008, https://doi.org/10.1016/ j.ecolmodel.2009.04.050.

Goodin, W. R., G. J. McRa, and J. H. Seinfeld, 1979: A comparison of interpolation methods for sparse data: Application to wind and concentration fields. J. Appl. Meteor., 18, 761-771, https://doi.org/ 10.1175/1520-0450(1979)018<0761:ACOIMF $>2.0 . C O ; 2$.

Guo, Z., H. Pan, W. Fan, and X. Lv, 2017: Application of surface spline interpolation in inversion of bottom friction coefficients. J. Atmos. Oceanic Technol., 34, 2021-2028, https://doi.org/ 10.1175/JTECH-D-17-0012.1.

Krige, D. G., 1952: A statistical analysis of some of the borehole values in the Orange Free State Goldfield. J. Chem. Metall. Min. Soc. S. Afr., 53, 47-70.

Le Dimet, F.-X., and O. Talagrand, 1986: Variational algorithms for analysis and assimilation of meteorological observations: Theoretical aspects. Tellus, 38A, 97-110, https://doi.org/10.3402/ tellusa.v38i2.11706.
Li, X., C. Wang, W. Fan, and X. Lv, 2013: Optimization of the spatiotemporal parameters in a dynamical marine ecosystem model based on the adjoint assimilation. Math. Probl. Eng., 2013, 373540, https://doi.org/10.1155/2013/373540.

Liu, Y., J. Yu, Y. Shen, and X. Lv, 2016: A modified interpolation method for surface total nitrogen in the Bohai Sea. J. Atmos. Oceanic Technol., 33, 1509-1517, https://doi.org/10.1175/ JTECH-D-15-0250.1.

__, Y. Shen, X. Lv, and Q. Liu, 2017: Numeric modelling and risk assessment of pollutions in the Chinese Bohai Sea. Sci. China Earth Sci., 60, 1546-1557, https://doi.org/10.1007/ s11430-016-9062-y.

Lu, X. Q., and J. C. Zhang, 2006: Numerical study on spatially varying bottom friction coefficient of a $2 \mathrm{D}$ tidal model with adjoint method. Cont. Shelf Res., 26, 1905-1923, https://doi.org/ 10.1016/j.csr.2006.06.007.

Meissa, B., and D. Gascuel, 2015: Overfishing of marine resources: Some lessons from the assessment of demersal stocks off Mauritania. ICES J. Mar. Sci., 72, 414-427, https://doi.org/ 10.1093/icesjms/fsu144.

Michalak, A. M., and P. K. Kitanidis, 2004: Estimation of historical groundwater contaminant distribution using the adjoint state method applied to geostatistical inverse modeling. Water Resour. Res., 40, W08302, https://doi.org/10.1029/ 2004WR003214.

Milnes, E., and P. Perrochet, 2007: Simultaneous identification of a single pollution point-source location and contamination time under known flow field conditions. Adv. Water Resour., 30, 2439-2446, https://doi.org/10.1016/j.advwatres.2007.05.013.

Navon, I. M., 1998: Practical and theoretical aspects of adjoint parameter estimation and identifiability in meteorology and oceanography. Dyn. Atmos. Oceans, 27, 55-79, https://doi.org/ 10.1016/S0377-0265(97)00032-8.

Neupauer, R. M., and J. L. Wilson, 1999: Adjoint method for obtaining backward-in-time location and travel time probabilities of a conservative groundwater contaminant. Water Resour. Res., 35, 3389-3398, https://doi.org/10.1029/1999WR900190.

— , and — 2005: Backward probability model using multiple observations of contamination to identify groundwater contamination sources at the Massachusetts Military Reservation. Water Resour. Res., 41, W02015, https://doi.org/10.1029/ 2003WR002974.

— , and R. Lin, 2006: Identifying sources of a conservative groundwater contaminant using backward probabilities conditioned on measured concentrations. Water Resour. Res., 42 , W03424, https://doi.org/10.1029/2005WR004115.

Oberai, A. A., N. H. Gokhale, and G. R. Feijóo, 2003: Solution of inverse problems in elasticity imaging using the adjoint method. Inverse Probl., 19, 297-313, https://doi.org/10.1088/ 0266-5611/19/2/304.

Øyvind, E., S. Eirik, J. K. Sundet, S. B. Dalsøren, I. S. A. Isaksen, T. F. Berglen, and G. Gravir, 2003: Emission from international sea transportation and environmental impact. J. Geophys. Res., 108, 4560, https://doi.org/10.1029/ 2002JD002898.

Pan, H., Z. Guo, and X. Lv, 2017: Inversion of tidal open boundary conditions of the M2 constituent in the Bohai and Yellow Seas. J. Atmos. Oceanic Technol., 34, 1661-1672, https://doi.org/ 10.1175/JTECH-D-16-0238.1.

Perrin, F., J. Pernier, O. Bertnard, M. Giard, and J. Echallier, 1987: Mapping of scalp potentials by surface spline interpolation. Electroencephalogr. Clin. Neurophysiol., 66, 75-81, https:// doi.org/10.1016/0013-4694(87)90141-6. 
Shen, Y., C. Wang, Y. Wang, L. V. Xianqing, and X. Lv, 2015: Inversion study on pollutant discharges in the Bohai Sea with the adjoint method. J. Ocean Univ. China, 14, 941-950, https:// doi.org/10.1007/s11802-015-2501-8.

Sidorovskaia, N. A., A. S. Ackleh, C. O. Tiemann, B. Ma, J. W. Ioup, and G. E. Ioup, 2016: Passive acoustic monitoring of the environmental impact of oil exploration on marine mammals in the Gulf of Mexico. Adv. Exp. Med. Biol., 875, 1007-1014, https://doi.org/10.1007/978-1-4939-2981-8_125.

Smedstad, O. M., and J. J. O'Brien, 1991: Variational data assimilation and parameter estimation in an equatorial Pacific Ocean model. Prog. Oceanogr., 26, 179-241, https://doi.org/ 10.1016/0079-6611(91)90002-4.

Snowden, R. J., and I. K. E. Ekweozor, 1987: The impact of a minor oil spillage in the estuarine Niger delta. Mar. Pollut. Bull., 18, 595-599, https://doi.org/10.1016/0025-326X(87)90279-7.

Sun, A. Y., S. L. Painter, and G. W. Wittmeyer, 2006: A constrained robust least squares approach for contaminant release history identification. Water Resour. Res., 42, W04414, https://doi.org/ 10.1029/2005WR004312.

Unser, M., A. Aldroubi, and M. Eden, 1991: Fast B-spline transforms for continuous image representation and interpolation. IEEE Trans. Pattern Anal. Mach. Intell., 13, 277-285, https:// doi.org/10.1109/34.75515.

Walsh, J. J., and Coauthors, 2015: A simulation analysis of the plankton fate of the Deepwater Horizon oil spills. Cont. Shelf Res., 107, 50-68, https://doi.org/10.1016/j.csr.2015.07.002.

_ J. J. Lenes, B. Darrow, A. Parks, and R. H. Weisberg, 2016: Impacts of combined overfishing and oil spills on the plankton trophodynamics of the West Florida shelf over the last half century of 1965-2011: A two-dimensional simulation analysis of biotic state transitions, from a zooplankton- to a bacterioplankton-modulated ecosystem. Cont. Shelf Res., 116, 54-73, https://doi.org/10.1016/j.csr.2016.01.007.

Wang, C., X. Li, and X. Lv, 2013: Numerical study on initial field of pollution in the Bohai Sea with an adjoint method. Math. Probl. Eng., 2013, 104591,https://doi.org/10.1155/2013/104591.

Wei, L., Z. Hu, L. Dong, and W. Zhao, 2015: A damage assessment model of oil spill accident combining historical data and satellite remote sensing information: A case study in Penglai 19-3 oil spill accident of China. Mar. Pollut. Bull., 91, 258-271, https://doi.org/10.1016/j.marpolbul.2014.11.036.

Wijnands, J. S., G. Qian, and Y. Kuleshov, 2016: Spline-based modelling of near-surface wind speeds in tropical cyclones.
Appl. Math. Model., 40, 8685-8707, https://doi.org/10.1016/ j.apm.2016.05.013.

Xing, Q., R. Meng, M. Lou, L. Bing, and X. Liu, 2015: Remote sensing of ships and offshore oil platforms and mapping the marine oil spill risk source in the Bohai Sea. Aquat. Procedia, 3, 127-132, https://doi.org/10.1016/j.aqpro.2015.02.236.

Xu, Q., C.-J. Qiu, and J.-X. Yu, 1994: Adjoint-method retrievals of low-altitude wind fields from single-Doppler wind data. J. Atmos. Oceanic Technol., 11, 579-585, https://doi.org/ 10.1175/1520-0426(1994)011<0579:AMROLA>2.0.CO;2.

Yang, G. Z., and N. Zabaras, 1998: The adjoint method for an inverse design problem in the directional solidification of binary alloys. J. Comput. Phys., 140, 432-452, https://doi.org/10.1006/ jcph.1998.5893.

Yao, Z. G., R. Y. He, X. W. Bao, D. X. Wu, and J. Song, 2012: M2 tidal dynamics in Bohai and Yellow Seas: A hybrid data assimilative modeling study. Ocean Dyn., 62, 753-769, https:// doi.org/10.1007/s10236-011-0517-1.

Yu, L., and J. J. O'Brien, 1992: On the initial condition in parameter estimation. J. Phys. Oceanogr., 22, 1361-1364, https://doi.org/ 10.1175/1520-0485(1992)022<1361:OTICIP > 2.0.CO;2.

Zanini, A., and A. D. Woodbury, 2016: Contaminant source reconstruction by empirical Bayes and Akaike's Bayesian information criterion. J. Contam. Hydrol., 185-186, 74-86, https://doi.org/10.1016/j.jconhyd.2016.01.006.

Zhang, J., and X. Lu, 2008: Parameter estimation for a threedimensional numerical barotropic tidal model with adjoint method. Int. J. Numer. Methods Fluids, 57, 47-92, https://doi.org/ 10.1002/fld.1620.

Zhang, X., and M. Huang, 2017: Ensemble-based release estimation for accidental river pollution with known source position. J. Hazard. Mater., 333,99-108, https://doi.org/10.1016/ j.jhazmat.2017.03.028.

—, J. G. Chen, G. F. Su, and H. Yuan, 2013: Study on source inversion technology for nuclear accidents based on Gaussian puff model and EnKF. Proc. 10th Int. ISCRAM Conf., BadenBaden, Germany, International Association for Information Systems for Crisis Response and Management, 634-639.

Zhang, Z., Y. Zhong, J. Tian, Q. Yang, and W. Zhao, 2014: Estimation of eddy heat transport in the global ocean from Argo data. Acta Oceanol. Sin., 33, 42-47, https://doi.org/10.1007/s13131-014-0421-x.

Zhao, P., and W. Jiang, 2011: A numerical study of storm surges caused by cold-air outbreaks in the Bohai Sea. Nat. Hazards, 59, 1-15, https://doi.org/10.1007/s11069-010-9690-7. 